\title{
Lesch-nyhan syndrome, a disease that could be easily missed: case series
}

\begin{abstract}
Purpose: Lesch-Nyhan syndrome (LNS) is an X-linked recessive disorder of purine metabolism with a defect in the activity of hypoxanthine-guanine phosphoribosyl transferase (HPRT). The aim of this study was to describe 2 cases of LNS, who were very difficult to be diagnosed.

Methods: Two cases of LNS were described: 6-month-old and 13-month-old children with delayed development, increased muscle tone, and kidney problem (hydronephrosis and nephrocalcinosis respectively).

Results: Diagnosis of LNS can be missed because 1) it is a very rare disease, 2) the usual laboratory serum uric acid level is based on adult men, not children, 3)some patients may only have hyperuricosuria, not hyperuricemia 4) clinical manifestations of LNS vary at different ages, 5) neuroimaging cannot help diagnose LNS, and 6) normal result of HPRT1 gene cannot rule out the diagnosis of LNS, and an analysis of HPRT activity is further needed in this case.
\end{abstract}

Conclusion: Urine uric acid/creatinine ratio should be checked in children with delayed development, with consideration for the above findings.
Volume 3 Issue 4 - 2018

\author{
Eun Jae Ko,' In Young Sung, ${ }^{2}$ Beom Hee Lee, ${ }^{3}$ \\ Han-Wook Yoo ${ }^{3}$ \\ 'Department of Physical Medicine and Rehabilitation, Ulsan \\ University Hospital, Korea \\ ${ }^{2}$ Department of Rehabilitation Medicine, University of Ulsan \\ College of Medicine, Korea \\ ${ }^{3}$ Department of Pediatrics, University of Ulsan College of \\ Medicine, Korea

\begin{abstract}
Correspondence: In Young Sung, Professor Department of Rehabilitation Medicine, Asan Medical Center, University of Ulsan College of Medicine, 88, Olympic-ro 43-gil, Songpa-gu, Seoul, 05505, Republic of Korea. Tel +82-2-3010-3792 Email iysung@amc.seoul.kr
\end{abstract}

Received: July 26, 2018 | Published: August 02, 2018

Keywords: lesch nyhan syndrome, children, developmental delay, hyperuricosuria, inborn errors of metabolism

\section{Introduction}

Lesch-Nyhan syndrome (LNS) is an X-linked recessive disorder of purine metabolism by mutation in Xq26 with a prevalence of $1: 100,000$ to $1: 380,000,{ }^{1,2}$ and there is a defect in the activity of the enzyme hypoxanthine-guanine phosphoribosyl transferase (HPRT). ${ }^{3}$ It was first described at John Hopkins Hospital in 1964 by Michael Lesch and William Nyhan who encountered two brothers with unusual symptoms: severe retardation of motor development, choreoathetosis, dystonia, crystals in the urine, and self-mutilation. ${ }^{1}$

Patients with LNS show hyperuricemia-related, neurologic, and behavioral features..$^{3-5}$ These symptoms may manifest at different periods. Furthermore, the phenotype of LNS varies and depends on the level of HPRT activity. Classic LNS is observed with a level of HPRT activity $<2 \%$, which involves significant motor and neurocognitive defects and the characteristic behavior of self-mutilation. The mildest phenotype of LNS variant is HPRT-related hyperuricemia with the level of HPRT activity $>8 \%$, and only hyperuricemia manifests. The intermediate phenotype is HPRT-related neurological dysfunction with $2-8 \%$ level of HPRT activity, and problems related to uric acid, along with varying degrees of motor, neurocognitive, and behavior problems. ${ }^{6}$ When the patient shows characteristic features of LNS, it is relatively easy to diagnose the disease. However, if a patient is very young and only shows mild symptoms of LNS, a diagnosis of LNS may be challenging. We described 2 cases of LNS: 6-month-old and 13-month-old children with delayed development, increased muscle tone, and kidney problem (hydronephrosis and nephrocalcinosis respectively), who were very difficult to be diagnosed.

\section{Clinical Presentation}

\section{Case I}

A 6-month-old child visited the outpatient clinic for delayed development. He was born by caesarean section at the gestational age of $38+4$ weeks and weighted $2600 \mathrm{~g}$. There was no abnormal family history. On his fetal ultrasonography, both hydronephrosis were observed, and he was followed up with ultrasonography after birth; no definite change was observed. His sucking power was relatively weak, but oral feeding was possible. At 6 months old, he had a height and weight of $70.2 \mathrm{~cm}$ (70 percentile) and $8.5 \mathrm{~kg}$ (50 percentile), respectively, with a head circumference of $45 \mathrm{~cm}$ (70 percentile). He could make eye contact with other people, smile, babble, and hold objects in his hand. However, he could not control his neck perfectly or roll his body. He showed head lagging with traction reaction, and he could hold his head up to $45^{\circ}$ in prone position. His sitting balance was poor, and he had no facial dysmorphism. He showed no primitive reflexes, and increased muscle tone in the bilateral upper and lower extremities were observed. Deep tendon reflexes were hyperactive, and he neither had ankle clonus nor Babinski's sign.

Results of a Denver Developmental Screening Test performed at 6 months were as follows: personal-social, 3-4 months; fine motoradaptive, 4-6 months; language, 6 months; and gross motor, 2 months. Magnetic resonance imaging (MRI) of the brain at 6 months old showed mild, diffuse thinning of the corpus callosum, mildly decreased white matter volume with enlarged extra-axial cerebrospinal fluid space, and delayed myelination (Figure 1). Since the development of gross motor skill was poorer than other categories of development and hypertonicity was observed in physical examination, MRI of the cervical spine was performed and showed no abnormal finding.

Tandem mass spectrometry screening, chromosome analysis, the test of microdeletion syndrome, amino acid analysis, and organic acid analysis in urine showed nonspecific findings. An increased uric acid/ creatinine ratio in the urine $(6.19 \mathrm{mg}$ of uric acid $/ \mathrm{mg}$ of creatinine) was observed, but the serum uric acid level showed mild elevation $(6.6 \mathrm{mg} / \mathrm{dL})$, which was first thought to be within the upper normal limit (normal range in our laboratory: $3-7 \mathrm{mg} / \mathrm{dL}$ ). Therefore, increased uric acid/creatinine ratio in the urine was considered associated with 
hydronephrosis. LNS was not considered in the differential diagnosis, because it is a very rare disease. However, orange crystals in the diaper were found during history taking, and the serum uric acid level was considered elevated based on the previous reference ${ }^{7}$ that a normal serum uric acid level is $3.6 \pm 0.9 \mathrm{mg} / \mathrm{dL}$ in children aged $<5$. With evidence of crystalluria and an increased uric acid level in the serum and urine, LNS was suspected. After consulting with the Department of Genetics, a genetic study was performed, and exon 1 deletion of the HPRT1 gene was observed, which is consistent with LNS Subsequently, treatment with oral allopurinol to control serum uric acid level and oral baclofen to control hypertonicity were initiated. Genetic study of his mother showed no abnormality, indicating the de novo mutation of HPRT1 gene in the patient.

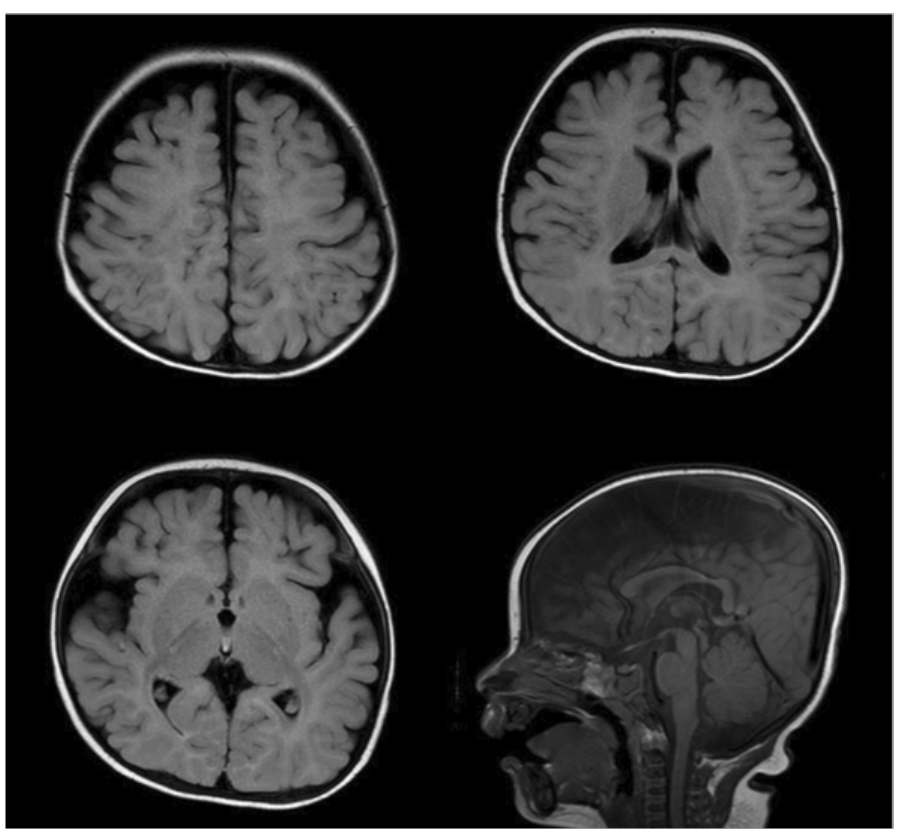

Figure I Brain magnetic resonance imaging showing mild, diffuse thinning of the corpus callosum, mildly decreased white matter volume with enlarged extra-axial cerebrospinal fluid space, and delayed myelination.

He underwent rehabilitation for delayed development; however, his development was very slow. Results of the Bayley Scale of Infant Development-II (BSID-II) performed at 12 months were as follows: mental and psychomotor development indices were $<50$, and developmental age was 5 months for cognition, 5 months for language, 2 months for fine motor skills, and 2-3 months for gross motor skills. Rolling was possible at the age of 13 months, but head control was still not perfect. At the age of 14 months, lip biting began, and serial follow up was decided for mouth guard.

He continues to receive treatments for LNS and to participate in the rehabilitation treatment program, and is followed up by Departments of Pediatric Rehabilitation Medicine, Pediatric Neurology, Genetics, Pediatric Nephrology, and Pediatric Odontology.

\section{Case 2}

A 13-month-old child visited the outpatient clinic for delayed development. He was born by caesarean section at the gestational age of $39+5$ weeks and weighted 2470 g. There was no abnormal family history. He could control his head at 3 months however, delayed development appeared after then: roll over at 11 months. At 13 months old, he had a height and weight of $78 \mathrm{~cm}$ (15 percentile) and $8.9 \mathrm{~kg}$ (10 percentile), respectively, with a head circumference of $44 \mathrm{~cm}$ (10 percentile). He could make eye contact with other people, smile, babble, and say mama and papa. However, he could not sit or creep. He showed no primitive reflexes, and increased muscle tone in the bilateral upper and lower extremities were observed. Deep tendon reflexes were hyperactive and he had equinovarus feet.

He had global developmental delay and the results of the BSIDII performed at 13 months were as follows: mental development index was 59; psychomotor development index was below 50; and developmental age was 6-7 months for cognition, 11-12 months for receptive language, 11 months for expressive language, 6-7 months for fine motor skills, and 5-6 months for gross motor skills. Magnetic resonance imaging (MRI) of the brain at 6 months old showed no abnormal finding.

Chromosome analysis showed nonspecific findings. Serum uric acid level showed mild elevation $(7.5 \mathrm{mg} / \mathrm{dL})$, and an increased uric acid/creatinine ratio in the urine $(4.49 \mathrm{mg}$ of uric acid $/ \mathrm{mg}$ of creatinine) was observed. On his kidney ultrasonography, multifocal intramedullary hyperechoic foci in both kidneys were observed, which present early findings of medullary nephrocalcinosis. With evidence of an increased uric acid level in the serum and urine, LNS was suspected, however, genetic study performed at the Department of Genetics showed no mutation detected in coding region of HPRT1 gene. Nevertheless, based on his clinical manifestations (delayed development, more in motor than in the cognitive category, and increased muscle tone) and laboratory findings (increased serum uric acid level and uric acid/creatinine ratio in the urine), LNS was still suspected. HPRT activity in erythrocytes was additionally analyzed, and it showed total deficient status $(0.0 \mathrm{micromol} / \mathrm{min} / \mathrm{g} \mathrm{Hb})$ (reference range: 3.5-6.0 micromol $/ \mathrm{min} / \mathrm{g} \mathrm{Hb}$ ), which is consistent with LNS. After diagnosed with LNS, treatment with oral allopurinol and oral baclofen were initiated. Ankle-foot-orthosis was recommended to control increased muscle tone at both ankles.

At the age of 15 months, sitting with two hand support and creeping were possible. He continues to receive treatments for LNS and to participate in the rehabilitation treatment program, and is followed up by Departments of Pediatric Rehabilitation Medicine, Genetics, and Pediatric Nephrology.

\section{Discussion}

LNS can be one of the causes of delayed development in children. As in the present case, accurate diagnosis of LNS is difficult when the main complaint is delayed development in a young child. In a previous literature review of patients with LNS, ${ }^{8}$ The average age at the time of case description was 9.5 years, with a range of 1 day to 33 years.

There are a few reasons for the possibility of missing the diagnosis of LNS. First, it is a very rare disease. ${ }^{2}$ Therefore, LNS is not commonly included in the differential diagnosis when a young child has delayed development.

Second, physicians should be careful with accepting a conclusion that the serum uric acid level is normal based on a laboratory whose norms were established for adult men in whom hyperuricemia is common. ${ }^{3}$ The normal serum uric acid level in children is different from that in adults. According to previous literature, it is $3.6 \pm 0.9 \mathrm{mg} /$ $\mathrm{dL}$ in children aged $<5$ years and $4.1 \pm 1.0 \mathrm{mg} / \mathrm{dL}$ in children aged between 5 and 10 years; [7] these led to the conclusion of an elevated serum uric acid level in the first case of this report. 
Third, although most patients present with hyperuricemia (uric acid level, usually 9-12 mg/dL), some patients with efficient uric acid excretion may only have hyperuricosuria, not hyperuricemia.[3-5] Therefore, not only uric acid level in the serum, but also that in the urine should be checked in a child with delayed development, and hyperuricosuria without hyperuricemia should not be missed as the sign of LNS. Hyperuricosuria can be detected by a 24-hour urine or spot urine sample. In a 24-hour sample, patients with LNS generally excrete 40-69 mg of uric acid per $\mathrm{kg}$ of body weight per day, compared to $18 \mathrm{mg}$ in normal children. ${ }^{4}$ However, microorganisms may consume purines, including uric acid, in a 24-hour urine sample, resulting in an inaccurate result. In contrast, a spot urine sample is more convenient to collect and analyze promptly for the uric acid/creatinine ratio. Three to $4 \mathrm{mg}$ of uric acid/ $\mathrm{mg}$ of creatinine is observed in patients with LNS compared to $<1$ in normal children older than 1 year. ${ }^{3}$ The increased uric acid/creatinine ratio in the above cases was the key finding for us to suspect LNS. In our literature review, the finding of a serum uric acid level $>4-5 \mathrm{mg} / \mathrm{dL}$ and a urine uric acid/creatinine ratio of 3-4 is known to be highly suggestive of LNS. ${ }^{4}$

Fourth, clinical manifestations of LNS vary at different ages, and a child, especially a young child, may not have typical symptoms of LNS. Most patients with LNS seem normal at birth and during the first months of life, except a few cases with hypotonia, recurrent vomiting, and difficulty with secretions. ${ }^{4}$ Clinical manifestations of LNS vary and include hyperuricemia-related, neurologic, and behavioral problems. The first sign of the disease is orange sand in the diaper (crystalluria), and it allows early diagnosis before development of neurologic or behavioral features, but it is easily missed in the clinical field. A deficiency of the HPRT enzyme result in hyperuricemia, and the symptoms related to it include urinary tract calculi, urinary tract infection, chronic tophaceous gout, renal failure, and rarely acute gouty arthritis. Regarding neurologic symptoms, defective motor development is apparent between 6 and 12 months old, and patients fail to reach developmental milestones with poor head control.[3] Patients with classic LNS do not learn to walk, and most do not sit alone. Muscle tone is variable, including hypotonia and hypertonia. Involuntary movements are seen in almost all patients, usually between 12 and 18 months (range 1 month to 6 years), ${ }^{3,4,8}$ and pyramidal features are sometimes seen. Impaired mental development is also observed, but a few patients show normal or near normal intelligence. Dysarthria, apraxic discoordination of the lips and tongue, and dysfunction of ocular motor activity are sometimes seen. ${ }^{3}$ Behavioral features are integral to the disease, and self-mutilation occurs in all patients with classic LNS, ${ }^{9}$ usually between 2 and 4 years, with the most characteristic features of self-destructive biting of the lips and fingers. Sometimes head banging and mutilation of the eyes and legs also appears. ${ }^{4}$ There is a tendency for them to improve with age and the symptoms to disappear by the end of the first decade of life. The differential diagnosis of self-injury includes the De Lange syndrome, dysautonomia, fragile $\mathrm{X}$ syndrome, Prader-Willi syndrome, and Smith-Magenis syndrome. ${ }^{3}$ The most distinguishing features of LNS appear after 12 months (12-18 months for involuntary movements and 2-4 years for self-mutilation on average); therefore it is difficult to diagnose LNS around 6 months.

Fifth, neuroimaging including brain computed tomography and MRI cannot help diagnose LNS, since most of the results are normal, except in a few cases with brain atrophy. ${ }^{8}$

Sixth, normal result of HPRT1 gene cannot rule out the diagnosis of LNS, and an analysis of HPRT activity is needed in this case. There are a few methods in diagnosing LNS. First method, which is an analysis of HPRT activity in erythrocytes,[10] has been used since the report by Seegmiller et al., ${ }^{11}$ in 1967, which showed the enzymatic activity of HPRT approaches zero in the erythrocytes of patients with classic LNS. Simple testing of enzyme activity has a limitation in carrier detection, because activity in heterozygotes is normal. ${ }^{12}$ Second method is an analysis of mutation of the HPRT1 gene, which is located on the long arm of the X chromosome (Xq26.1) and encodes human HPRT. It is about 44-kb long, composed of nine exons and transcribed into a 1.6-kb mRNA which encodes a protein of 218 amino acids. ${ }^{13}$ It has strengths in carrier and prenatal diagnosis. ${ }^{5}$ However, normal result of HPRT1 gene cannot rule out the diagnosis of LNS, as in the second case of this report. There were some previous studies ${ }^{14-16}$ which reported LNS patients with enzyme proven deficiency of HPRT and no mutation detectable in the HPRT1 gene, in whom decreased mRNA expression of HPRT were found. It suggests that the deficiency of the enzyme might be caused by mutations in regulating elements that determine gene expression, such as promoter and intronic sequences. There is a possibility that decreased mRNA expression of HPRT might be found in the second case of this report, which was not further investigated.

There is no curative treatment for LNS, but symptomatic treatments are possible. Allopurinol, which is a xanthine oxidase inhibitor, is effective in reducing the uric acid concentration and alleviating all of its direct clinical consequences. However, it has no effect on the development of the neurobehavioral symptoms of LNS. ${ }^{10}$ To treat neurologic symptoms, rehabilitation and drugs such as muscle relaxants, benzodiazepine, and carbamazepine can be used. ${ }^{5}$ There is no effective treatment for self-mutilation. Encouraging purposeful activity is recommended to replace self-injurious behavior, and physical restraints and flexible arm splints are used to decrease finger biting. Sometimes, the selective removal of teeth is performed reluctantly. ${ }^{3}$ Treatments with deep brain stimulation ${ }^{17}$ and S-Adenylmethionine ${ }^{18}$ has been reported, and these are promising methods for treating self-mutilating behavior associated with LNS. Additionally, pharmacologic approaches to therapy based on the neurotransmitter imbalance and gene therapy have not yet been successful in vivo, but this is a promising direction.

With active medical treatment, patients with LNS can survive up to their 20-30s, but renal failure occurs in 10 years without treatment. ${ }^{5}$ Causes of death include complications of renal failure and aspiration pneumonia; however, some patients die suddenly without known causes. ${ }^{3,5}$ The early diagnosis can prevent renal complications; therefore, finding clues for the disease and making an early diagnosis of LNS are necessary.

In conclusion, LNS can be one of the causes in a child with delayed development, and early detection of LNS is challenging when there are only mild symptoms without typical symptoms. The urine uric acid/creatinine ratio should be checked in children with delayed development, and physicians should be careful in interpreting a serum uric acid level based on the child's age. When there is hyperuricosuria with or without hyperuricemia, LNS should be suspected, and to confirm the diagnosis, both analyses of HPRT activity and HPRT1 gene are sometimes needed. Furthermore, a history of orange crystals in children's diapers should not be missed. The early diagnosis and treatment of LNS would definitely reduce renal complications, leading to longer life expectancy. 


\section{Acknowledgements}

None.

\section{Conflict of interest}

Author declares that there is no conflicts of interest.

\section{References}

1. Lesch M, Nyhan WL. A familial disorder of uric acid metabolism and central nervous system function. Am J Med. 1964;36:561-570.

2. Tewari N, Mathur VP, Sardana D, et al. Lesch-Nyhan syndrome: The saga of metabolic abnormalities and self-injurious behavior. Intractable Rare Dis Res. 2017;6(1):65-68.

3. Nyhan WL, Barshop BA, Al-Aqeel AI. Atlas of inherited metabolic disease. 3rd ed. London: Hodder Arnold. 2012.

4. Lyon G, Kolodny EH, Pastores GM. Neurology of hereditary metabolic diseases of children. 3rd ed. New York (NY): McGraw-Hill Education. 2006.

5. Yoo HW. Inherited metabolic disease. Seoul: Korea Medical Book Pubilishing Company. 2016.

6. Fu R, Sutcliffe D, Zhao H, et al. Clinical severity in Lesch-Nyhan disease: the role of residual enzyme and compensatory pathways. $\mathrm{Mol}$ Genet Metab. 2015;114(1):55-61.

7. Wilcox WD. Abnormal serum uric acid levels in children. J Pediatr. 1996;128(6):731-741.

8. Jinnah HA, Visser JE, Harris JC, et al. Delineation of the motor disorder of Lesch-Nyhan disease. Brain. 2006;129(5):1201-1217.
9. Christie R, Bay C, Kaufman IA, et al. Lesch-Nyhan disease: clinical experience with nineteen patients. Dev Med Child Neurol. 1982;24(3):293-306.

10. Nyhan WL. Lesch-Nyhan disease. J Hist Neurosci. 2005;14:1-10.

11. Seegmiller JE, Rosenbloom FM, Kelley WN. Enzyme defect associated with a sex-linked human neurological disorder and excessive purine synthesis. Science. 1967;155(3770):1682-1684.

12. Francke U, Felsenstein J, Gartler SM, et al. The occurrence of new mutants in the X-linked recessive Lesch-Nyhan disease. Am J Hum Genet. 1976;28(2):123-137.

13. Patel PI, Framson PE, Caskey CT, et al. Fine structure of the human hypoxanthine phosphoribosyl transferase gene. Mol Cell Biol. 1986;6(2):393-403.

14. Garcia MG, Torres RJ, Prior C, et al. Normal HPRT coding region in complete and partial HPRT deficiency. Mol Genet Metab. 2008;94(2):167-172.

15. Dawson PA, Gordon RB, Keough DT, et al. Normal HPRT coding region in a male with gout due to HPRT deficiency. Mol Genet Metab. $2005 ; 85(1): 78-80$.

16. Nguyen KV, Naviaux RK, Paik KK, et al. Lesch-Nyhan syndrome: mRNA expression of HPRT in patients with enzyme proven deficiency of HPRT and normal HPRT coding region of the DNA. Mol Genet Metab. 2012;106(4):498-501.

17. Bell S, Kolobova I, Crapper L, et al. Lesch-Nyhan syndrome: models, theories, and therapies. Mol Syndromol. 2016;7(6):302-311.

18. Lauber M, Plecko B, Pfiffner M, et al. The effect of s-adenosylmethionine on self-mutilation in a patient with Lesch-Nyhan disease. JIMD Rep. 2017;32:51-57. 